\title{
Resonant Auger electron-ion-coincidence spectroscopy of $N$-methyltrifluoroacetamide: Site-specific fragmentation studies
}

\author{
Peter Salén $\odot,{ }^{1, *}$ Luca Schio $\odot,{ }^{2,3}$ Robert Richter, ${ }^{4}$ Michele Alagia $\odot,{ }^{2}$ Stefano Stranges $\odot,{ }^{2,5}$ and Vitali Zhaunerchyk ${ }^{6, \dagger}$ \\ ${ }^{1}$ FREIA Laboratory, Department of Physics and Astronomy, Uppsala University, 75120 Uppsala, Sweden \\ ${ }^{2}$ TASC Laboratory, IOM-CNR, 34149 Basovizza, Trieste, Italy \\ ${ }^{3}$ Dipartimento di Scienze di Base e Applicate per l'Ingegneria, Sapienza Università di Roma, 00185 Rome, Italy \\ ${ }^{4}$ Elettra Sincrotrone Trieste, 34149 Basovizza, Trieste, Italy \\ ${ }^{5}$ Dipartimento di Chimica e Tecnologie del Farmaco, Sapienza Università di Roma, 00185 Roma, Italy \\ ${ }^{6}$ Department of Physics, University of Gothenburg, 41296 Gothenburg, Sweden
}

(Received 24 April 2020; accepted 27 August 2020; published 17 September 2020)

\begin{abstract}
We present a theoretical and experimental study of the electronic structure of $N$-methyltrifluoroacetamide (FNMA) and examine the resonant Auger (RA) decay upon site- and state-specific excitation at the C $1 s, \mathrm{~N}$ $1 s$, and $\mathrm{O} 1 s$ edges. Based on the calculated energies for cationic states of $1 h$ and $2 h-1 p$ configurations, we assign the peaks of recorded VUV photoelectron and RA spectra. Specifically, we identify the spectral features associated with participator and spectator RA decay. To aid in a deeper understanding of previous observations of limited site-specific bond breaking in FNMA, the influence of the site-specific excitation and the character of the Auger decay on the fragmentation is investigated using Auger electron ion coincidence experiments. Moreover, to investigate the potentially different capacity for selective bond cleavage of participator and spectator Auger states, we apply this technique to the spectator Auger state with the main electronic configuration of $\left(31 a^{\prime}\right)^{-1}\left(32 a^{\prime \prime}\right)^{-1}\left(34 a^{\prime \prime}\right)^{1}$, which is well resolved in the experimental spectrum. Finally, the influence of the molecular excess energy and the electronic character of the RA final states on fragmentation is addressed. Based on our findings, we argue that the excess energy the FNMA molecule gains upon RA decay is the dominant factor determining the fragmentation pattern.
\end{abstract}

DOI: 10.1103/PhysRevA.102.032817

\section{INTRODUCTION}

The excitation of core electrons by $\mathrm{x}$-ray radiation is site specific, i.e., the same atomic species, or different elements, at distinct molecular sites can be targeted. It thus permits the effect of the local character of the excitation to be investigated. In resonant Auger (RA) spectroscopy the kinetic energy of the electrons emitted via Auger decay, following resonant core excitation, is recorded. This enables a site- and state-specific study of the Auger process and provides information about, e.g., Auger decay rates, the electronic structure, and orbital characteristics of the molecule [1-4]. Site-specific studies initiated by core ionization require coincident detection of the ionized core electron and subsequent products (normal Auger electrons or fragments) in order to infer which site has initially been core ionized. By contrast, the site specificity is inherent to core excitation, i.e., by tuning the x-ray photon

\footnotetext{
*peter.salen@physics.uu.se

†vitali.zhaunerchyk@physics.gu.se
}

Published by the American Physical Society under the terms of the Creative Commons Attribution 4.0 International license. Further distribution of this work must maintain attribution to the author(s) and the published article's title, journal citation, and DOI. Funded by Bibsam. energy, a particular core-excited site can be selected. Another essential difference between core ionization and excitation is that normal Auger decay following core ionization leads to a doubly charged, or higher, final state, while RA decay typically results in a singly charged molecule, although higher charge states are also possible for the RA process.

The site-specific excitation has been proposed as a tool for selectively breaking particular bonds of a molecule. A number of studies have been performed on this topic using both resonant core excitation [5-17] and core ionization [17-31], showing varying degree of selectivity. A recent study [31] on core ionization of molecules has shown only a small degree of selectivity which was explained by the delocalized character of the valence orbitals. As the excitation and fragmentation is intermediated by the Auger decay, the latter presumably plays a decisive role for site-specific fragmentation. The RA process can be divided into two types with conceivably distinct potential for such selective bond breaking. (1) In the participator RA decay the core electron, which is excited to an unoccupied molecular orbital, participates in the subsequent Auger process, in which the core hole is filled and an electron is ejected. The final-state electronic configuration is of one-hole (1h) character. If the valence-hole orbital is of, e.g., strongly bonding character at a specific bond, then one may expect that bond to weaken and be more prone to breaking. The Auger decay favors the formation of final states with valence holes located on molecular orbitals that overlap with that of the 


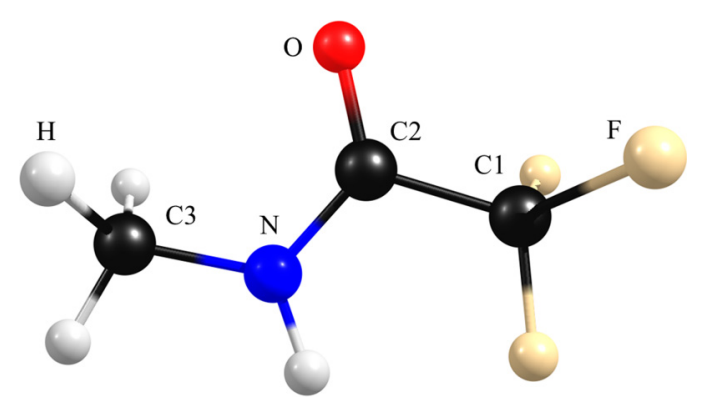

FIG. 1. FNMA geometry optimized at the CAM-B3LYP-D3BJ aug-cc-pVTZ level of theory.

core hole [1]. Hence, there is a certain selectivity in removing electrons from orbitals localized around the initial site of core excitation. (2) In the spectator RA decay the core electron is excited to an unoccupied molecular orbital and stays there, acting as a spectator, during the Auger decay. The final-state electronic configuration is of two-hole-one-particle $(2 h-1 p)$ type. If the spectator electron is placed in an electronic orbital with a strongly antibonding character at a specific bond, the chance can increase to selectively break that bond. This suggests that the spectator Auger decay has a higher potential for selective bond breaking compared with the participator counterpart, since empty (virtual) molecular orbitals are more often of antibonding character than occupied orbitals.

Coincidence measurements of the Auger electrons and ion fragments enable correlating a particular Auger final state with fragmentation channels through which the molecule breaks up. Such experiments on $2 \mathrm{Br}$ pyrimidine [13] have demonstrated a correlation between the orbital where the valence hole is created upon RA decay and the preferred fragmentation channel. More specifically, a vacancy formed in an electronic orbital with bonding character at a certain bond resulted in a higher probability to break that bond. Based on such observations, it was proposed that for $2 \mathrm{Br}$ pyrimidine the selectivity in bond breaking was determined by the final-electronic-state populations, i.e., the Auger populations, resulting from different inner-shell excitations.

Although the Auger population is suggested to be a key factor in the selectivity of bond breaking resulting from both core excitation [13] and core ionization [28], the actual mechanism that leads to the selectivity may vary. Some studies point to the character of the electronic states populated after Auger decay as the determining component [13,31], while others associate it with the internal energy distribution of the molecule [27,28]. In addition to the role played by the Auger population for the selectivity, the core-ionization-induced nuclear motion occurring before the Auger decay has been proposed to be a significant factor in some cases [24,30]. A further discussion of previous studies on site-dependent fragmentation effects can be found in Ref. [28].

In this paper we study the electronic structure and RA decay of the peptide model system $N$-methyltrifluoroacetamide (FNMA), shown in Fig. 1, in order to obtain a further understanding of site- and state-specific fragmentation. With the help of quantum chemical calculations, we find final-state energies, which permits the assignment of spectral features in the recorded vacuum ultraviolet (VUV) photoelectron spectrum (PES) and in RA spectra upon C $1 s^{-}, \mathrm{N} 1 s^{-}$, and $\mathrm{O} 1 s$-edge excitation. Using Auger electron-ion-coincidence techniques, we investigate how the fragmentation of the molecule depends on the site- and state-specific excitation and on the properties of the Auger decay. Thus, we are able to explain previous results [32] that have indicated site-specific bond breaking in FNMA. Moreover, the influence of the Auger decay type, i.e., spectator or participator decay, on the selectivity of bond breaking is addressed. We also examine the significance of the molecular excess energy on the fragmentation.

\section{METHODS}

\section{A. Experimental approach and data analysis}

The experimental measurements have been performed at the GasPhase beamline of the Elettra synchrotron facility [33]. The soft $x$ rays are generated in a variable-gap undulator that allows tuning of the output photon energy. A monochromator, located downstream the beamline, enables a spectral width below the natural linewidth. FNMA was acquired commercially from Sigma-Aldrich. The FNMA sample was heated to approximately $35^{\circ} \mathrm{C}$ and introduced into the interaction region effusively, where it was irradiated with the synchrotron light. The near-edge $\mathrm{x}$-ray-absorption fine-structure (NEXAFS) spectra at the C $1 s, \mathrm{~N} 1 s$, and $\mathrm{O} 1 s$ edges were measured by scanning soft-X-ray photon energies and monitoring ion intensity with a time-of-flight mass spectrometer collecting all ions. The ion intensity was normalized to photon flux. The measured NEXAFS spectra were calibrated to those previously reported in Ref. [32]. The VUV PES and RA spectra at different photon energies were recorded with a commercial hemispherical electron energy analyzer (VG220i). Ions in coincidence with electrons were measured with a homebuilt ion time-of-flight spectrometer [34]. The VUV PES spectrum was calibrated to the cation ground state of water (the cationic state associated with ionization of the highest occupied molecular orbital) [35], using residual water. Below-resonance contributions were subtracted from the RA spectra, except where indicated. The acquisition time was identical for the resonant and below-resonant spectra; thus only photon flux was used for normalization of their relative intensities before subtraction. Resonant Auger spectra with below-resonance contributions subtracted were smoothed using Savitzky-Golay filtering. The experimental energy resolution of each presented spectrum is given in the captions of Figs. 2-6 and 8. For the NEXAFS spectra, the energy resolution was estimated based on the slit widths of the beamline monochromator and for the electron spectra also the pass energy of the electron energy analyzer was taken into account.

\section{B. Theoretical approach}

The theoretical calculations presented in this paper have been performed with the GAUSSIAN software [36]. Under normal conditions FNMA predominantly adopts two trans conformers which can be superimposed by rotating the methyl group by $60^{\circ}$ [32]. These two conformers are very similar 


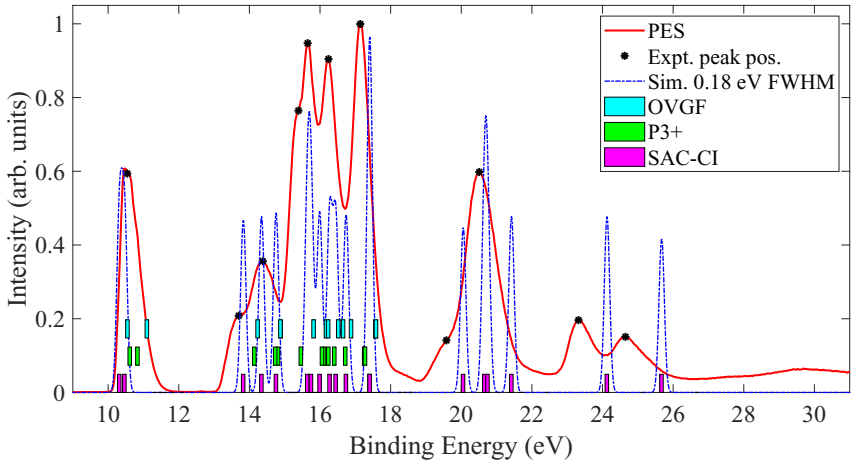

FIG. 2. The VUV PES measured at $h v=60 \mathrm{eV}$ is presented by the red curve. The black stars indicate estimated experimental peak positions. The VUV PES was simulated using peaks of Gaussian profile having a FWHM of $0.18 \mathrm{eV}$ (blue dash-dotted line), which corresponds to the estimated experimental energy resolution. The bars show the theoretical results from Table II. The simulated spectrum was produced using the ionization energies and intensities obtained with the SACCI method.

in structure as well as in energy and it was shown that their NEXAFS spectra are nearly identical [32]. We assume their VUV PES and RA spectra to be very similar as well and therefore in our theoretical calculations we consider only one of these conformers which is presented in Fig. 1. The geometry of this conformer was optimized at the CAM-B3LYP aug-ccpVTZ level of theory including D3BJ empirical dispersion.

Vertical ionization energies (VIEs) are calculated as the difference in electronic energies between ionized and neutral FNMA. The electronic energy of the ionized state is found by performing single-point energy calculations for the optimized structure of the neutral ground state. To investigate the consistency of different theoretical methods, similar calculations have been performed with other density functional theory (DFT) functionals as well as G4MP2 and second-order Moller-Plesset perturbation theory (MP2) ab initio method (Table I). For all methods, except G4MP2, the geometry of the neutral FNMA was optimized with the corresponding method. The G4MP2 calculations were performed for the CAM-B3LYP-D3BJ aug-cc-pVTZ optimized geometry.

Thermodynamic thresholds of different fragmentation channels (i.e., the minimum energy which is required for neutral FNMA to energetically access formation of certain fragments) have been calculated as the difference in enthalpies at $300 \mathrm{~K}$ between the corresponding fragments and the parent molecule. The enthalpies are calculated for FNMA and fragments optimized at the CAM-B3LYP-D3BJ aug-cc-pVTZ level of theory (Table IV).

To assign spectral features in VUV photoelectron spectra, two approaches are employed. The first approach is based on electron propagator theory (EPT) using the renormalized partial third-order approximation $(\mathrm{P} 3+)$ and the outer valence Green's function propagator (OVGF). The second approach involves the symmetry-adapted cluster configuration interaction (SACCI) with the SD-R method which includes singles and doubles excitation operators. With both approaches, calculations were performed for the CAM-B3LYP-D3BJ augcc-pVTZ optimized structure (Fig. 1). For the SACCI method
TABLE I. Vertical ionization energies of FNMA calculated with different methods. The values were obtained as the difference in electronic energy of the neutral ground state and the lowest energy ionic states of $A^{\prime}$ and $A^{\prime \prime}$ symmetry. The geometry of the neutral state was optimized with the different methods, while for the two ionic states the optimized geometry of the neutral was assumed. All the values are presented in $\mathrm{eV}$ units. In the methods designated with an asterisk no geometry optimization is performed and the CAM-B3LYP-D3BJ aug-cc-pVTZ optimized structure is used for energy calculations.

\begin{tabular}{lcc}
\hline \hline Method & $1^{2} A^{\prime \prime}$ & $1^{2} A^{\prime}$ \\
\hline CAM-B3LYP-D3BJ aug-cc-pVTZ & 10.552 & 10.568 \\
B3LYP-D3BJ aug-cc-pVTZ & 10.464 & 10.550 \\
$\omega$ B97XD aug-cc-pVTZ & 10.447 & 10.521 \\
M062X aug-cc-pVTZ & 10.721 & 10.796 \\
G4MP2* & 10.525 & 10.632 \\
MP2 aug-cc-pVTZ & 9.030 & 9.320 \\
EPT-P3+ aug-cc-pVTZ* & 10.611 & 10.830 \\
EPT-OVGF aug-cc-pVTZ* & 10.546 & 11.096 \\
SACCI aug-cc-pVTZ* & 9.930 & 10.111 \\
SACCI cc-pVTZ* & 10.315 & 10.460 \\
\hline \hline
\end{tabular}

the basis set of cc-pVTZ was used as it was shown to be more accurate for ionization energies [37]. The VIEs obtained with these methods are collected in Table II.

The spectral features in RA spectra are of two types: participator and spectator. Participator Auger peaks are associated with the same final states as those populated upon direct valence ionization and are described by the $1 \mathrm{~h}$ electronic configuration. Spectator states are more complex and are characterized by the $2 h-1 p$ configuration. The SACCISD-R method is not sufficient to adequately describe $2 h-1 p$ states and the SACCI-R-general method is implemented instead (Table III). The general-R method includes excitation operators up to sextuples.

\section{RESULTS AND DISCUSSION}

\section{A. VUV photoelectron spectra}

According to the self-consistent-field density population analysis, the electronic configuration of the FNMA ground state of $C_{s}$ symmetry is $\ldots\left(14 a^{\prime}\right)^{2}\left(15 a^{\prime}\right)^{2}\left(16 a^{\prime}\right)^{2}\left(17 a^{\prime}\right)^{2}$ $\left(18 a^{\prime \prime}\right)^{2}\left(19 a^{\prime}\right)^{2}\left(20 a^{\prime}\right)^{2}\left(21 a^{\prime \prime}\right)^{2}\left(22 a^{\prime}\right)^{2}\left(23 a^{\prime}\right)^{2}\left(24 a^{\prime \prime}\right)^{2}\left(25 a^{\prime}\right)^{2}$ $\left(26 a^{\prime \prime}\right)^{2}\left(27 a^{\prime \prime}\right)^{2}\left(28 a^{\prime}\right)^{2}\left(29 a^{\prime}\right)^{2}\left(30 a^{\prime \prime}\right)^{2}\left(31 a^{\prime}\right)^{2}\left(32 a^{\prime \prime}\right)^{2}\left(33 a^{\prime}\right)^{0}$ $\left(34 a^{\prime \prime}\right)^{0} \ldots$ The first two VIEs correspond to the removal of an electron from the $32 a^{\prime \prime}$ and $31 a^{\prime}$ orbitals, thus forming $1^{2} A^{\prime \prime}$ and $1^{2} A^{\prime}$ ionic states, respectively. The VIE values calculated with different methods are presented in Table I. Apart from the $a b$ initio MP2 method, the values calculated with the different DFT (CAM-B3LYP, B3LYP, $\omega$ B97XD, and M062X) functionals, G4MP2, EPT, and SACCI cc-pVTZ methods are very close to each other and generally agree well with the experimental value of $10.5 \pm 0.1 \mathrm{eV}$, obtained in the present work. This value was determined as the position of the lowest-energy peak in the VUV PES. The VIE values obtained with the SACCI theory are quite sensitive to the choice of basis set. In particular, the smaller basis set cc-pVTZ for the SACCI method is more accurate than aug-cc-pVTZ [37]. In light of this effect, all further SACCI calculations 
TABLE II. The VIEs of FNMA. The SACCI energies correspond to ionized states with $1 h$ main configurations and the expansion coefficients of different $1 h$ configurations are presented in parentheses. For the EPT methods MO refers to the ionized molecular orbital. All values are given in $\mathrm{eV}$ units.

\begin{tabular}{|c|c|c|c|c|c|c|c|}
\hline \multirow[b]{2}{*}{ Experiment } & \multicolumn{3}{|c|}{ SACCI-SD-R cc-pVTZ } & \multicolumn{2}{|c|}{$\begin{array}{c}\text { EPT-P3+ } \\
\text { aug-cc-pVTZ }\end{array}$} & \multicolumn{2}{|c|}{$\begin{array}{c}\text { EPT-OVGF } \\
\text { aug-cc-pVTZ }\end{array}$} \\
\hline & Energy & State & Main configuration & Energy & MO & Energy & $\mathrm{MO}$ \\
\hline \multirow[t]{2}{*}{10.5} & 10.315 & $1^{2} A^{\prime \prime}$ & $\left(32 a^{\prime \prime}\right)^{-1}(0.97)$ & 10.611 & $32 a^{\prime \prime}$ & 10.546 & $32 a^{\prime \prime}$ \\
\hline & 10.46 & $1^{2} A^{\prime}$ & $\left(31 a^{\prime}\right)^{-1}(-0.96)$ & 10.83 & $31 a^{\prime}$ & 11.096 & $31 a^{\prime}$ \\
\hline 13.7 & 13.823 & $2^{2} A^{\prime \prime}$ & $\left(30 a^{\prime \prime}\right)^{-1}(0.94)$ & 14.14 & $30 a^{\prime \prime}$ & 14.232 & $30 a^{\prime \prime}$ \\
\hline \multirow[t]{2}{*}{14.4} & 14.38 & $2^{2} A^{\prime}$ & $\left(28 a^{\prime}\right)^{-1}(0.95)$ & 14.73 & $28 a^{\prime}$ & 14.879 & $29 a^{\prime}$ \\
\hline & 14.751 & $3^{2} A^{\prime}$ & $\left(29 a^{\prime}\right)^{-1}(-0.94)$ & 14.812 & $29 a^{\prime}$ & 14.879 & $28 a^{\prime}$ \\
\hline 15.4 & 15.639 & $3^{2} A^{\prime \prime}$ & $\left(26 a^{\prime \prime}\right)^{-1}(0.97)$ & 15.456 & $26 a^{\prime \prime}$ & 15.817 & $26 a^{\prime \prime}$ \\
\hline \multirow[t]{2}{*}{15.6} & 15.744 & $4^{2} A^{\prime}$ & $\left(25 a^{\prime}\right)^{-1}(-0.89)$ & 16.055 & $25 a^{\prime}$ & 16.168 & $27 a^{\prime \prime}$ \\
\hline & 15.986 & $4^{2} A^{\prime \prime}$ & $\left(27 a^{\prime \prime}\right)^{-1}(0.66),\left(24 a^{\prime \prime}\right)^{-1}(-0.68)$ & 16.116 & $27 a^{\prime \prime}$ & 16.23 & $25 a^{\prime}$ \\
\hline \multirow[t]{3}{*}{16.3} & 16.269 & $5^{2} A^{\prime}$ & $\left(23 a^{\prime}\right)^{-1}(0.85)$ & 16.235 & $24 a^{\prime \prime}$ & 16.518 & $24 a^{\prime \prime}$ \\
\hline & 16.441 & $5^{2} A^{\prime \prime}$ & $\left(24 a^{\prime \prime}\right)^{-1}(0.69),\left(27 a^{\prime \prime}\right)^{-1}(0.67)$ & 16.404 & $23 a^{\prime}$ & 16.653 & $23 a^{\prime}$ \\
\hline & 16.733 & $6^{2} A^{\prime}$ & $\left(22 a^{\prime}\right)^{-1}(0.81)$ & 16.714 & $22 a^{\prime}$ & 16.878 & $22 a^{\prime}$ \\
\hline \multirow[t]{2}{*}{17.1} & 17.403 & $6^{2} A^{\prime \prime}$ & $\left(21 a^{\prime \prime}\right)^{-1}(-0.97)$ & 17.251 & $21 a^{\prime \prime}$ & 17.569 & $21 a^{\prime \prime}$ \\
\hline & 17.404 & $7^{2} A^{\prime}$ & $\left(20 a^{\prime}\right)^{-1}(0.96)$ & 17.274 & $20 a^{\prime}$ & 17.585 & $20 a^{\prime}$ \\
\hline 19.6 & 20.05 & $9^{2} A^{\prime}$ & $\left(19 a^{\prime}\right)^{-1}(0.93)$ & & & & \\
\hline \multirow[t]{3}{*}{20.5} & 20.653 & $7^{2} A^{\prime \prime}$ & $\left(18 a^{\prime \prime}\right)^{-1}(0.97)$ & & & & \\
\hline & 20.742 & $10^{2} A^{\prime}$ & $\left(17 a^{\prime}\right)^{-1}(0.89)$ & & & & \\
\hline & 21.417 & $11^{2} A^{\prime}$ & $\left(16 a^{\prime}\right)^{-1}(-0.96)$ & & & & \\
\hline 23.3 & 24.122 & $14^{2} A^{\prime}$ & $\left(15 a^{\prime}\right)^{-1}(-0.96)$ & & & & \\
\hline 24.7 & 25.67 & $\ldots^{2} A^{\prime}$ & $\left(14 a^{\prime}\right)^{-1}(0.90)$ & & & & \\
\hline
\end{tabular}

TABLE III. Vertical energies of the $2 h-1 p$ states of FNMA with respect to the neutral ground state obtained with the SACCI generalR cc-pVTZ method. The table lists configurations of the $2 h-1 p$ states with expansion coefficients $|C|>0.4$. The expansion coefficients are presented in parentheses. The electronic configurations are presented in the form $a^{-1} b^{-1} c^{1}$, where $a$ denotes the molecular orbital from which an electron is excited to an unoccupied molecular orbital $c$, and $b$ is an ionized molecular orbital. All values are given in $\mathrm{eV}$ units.

\begin{tabular}{lll}
\hline \hline State & Energy & \multicolumn{1}{c}{ Main configuration } \\
\hline $8^{2} A^{\prime}$ & 19.338 & $\left(31 a^{\prime}\right)^{-1}\left(32 a^{\prime \prime}\right)^{-1}\left(34 a^{\prime \prime}\right)^{1}(-0.99)$ \\
$8^{2} A^{\prime \prime}$ & 21.654 & $\left(32 a^{\prime \prime}\right)^{-1}\left(31 a^{\prime}\right)^{-1}\left(34 a^{\prime \prime}\right)^{1}(-0.54)$ \\
$12^{2} A^{\prime}$ & 21.712 & $\left(32 a^{\prime \prime}\right)^{-2}\left(34 a^{\prime \prime}\right)^{1}(-0.75)$ \\
& $\left(32 a^{\prime \prime}\right)^{-1}\left(31 a^{\prime}\right)^{-1}\left(34 a^{\prime \prime}\right)^{1}(-0.72)$ \\
$9^{2} A^{\prime \prime}$ & 22.392 & $\left(30 a^{\prime \prime}\right)^{-1}\left(31 a^{\prime}\right)^{-1}\left(34 a^{\prime \prime}\right)^{1}(-0.42)$ \\
& & $\left(31 a^{\prime}\right)^{-2}\left(34 a^{\prime \prime}\right)^{1}(-0.80)$ \\
$13^{2} A^{\prime}$ & 23.058 & $\left(28 a^{\prime}\right)^{-1}\left(32 a^{\prime \prime}\right)^{-1}\left(34 a^{\prime \prime}\right)^{1}(0.82)$ \\
& & $\left(31 a^{\prime}\right)^{-1}\left(30 a^{\prime \prime}\right)^{-1}\left(34 a^{\prime \prime}\right)^{1}(0.54)$ \\
$10^{2} A^{\prime \prime}$ & 24.217 & $\left(32 a^{\prime \prime}\right)^{-1}\left(28 a^{\prime}\right)^{-1}\left(34 a^{\prime \prime}\right)^{1}(0.47)$ \\
& & $\left(29 a^{\prime}\right)^{-1}\left(31 a^{\prime}\right)^{-1}\left(34 a^{\prime \prime}\right)^{1}(0.97)$ \\
$11^{2} A^{\prime \prime}$ & 24.463 & $\left(31 a^{\prime}\right)^{-1}\left(29 a^{\prime}\right)^{-1}\left(34 a^{\prime \prime}\right)^{1}(0.89)$ \\
& & $\left(28 a^{\prime}\right)^{-1}\left(31 a^{\prime}\right)^{-1}\left(34 a^{\prime \prime}\right)^{1}(-0.45)$ \\
& & $\left(29 a^{\prime}\right)^{-1}\left(31 a^{\prime}\right)^{-1}\left(34 a^{\prime \prime}\right)^{1}(0.40)$ \\
$15^{2} A^{\prime}$ & 24.588 & $\left(29 a^{\prime}\right)^{-1}\left(32 a^{\prime \prime}\right)^{-1}\left(34 a^{\prime \prime}\right)^{1}(0.66)$ \\
& & $\left(28 a^{\prime}\right)^{-1}\left(32 a^{\prime \prime}\right)^{-1}\left(34 a^{\prime \prime}\right)^{1}(-0.42)$ \\
$12^{2} A^{\prime \prime}$ & 24.729 & $\left(25 a^{\prime}\right)^{-1}\left(32 a^{\prime \prime}\right)^{-1}\left(34 a^{\prime \prime}\right)^{1}(-0.41)$ \\
$13^{2} A^{\prime \prime}$ & 24.760 & $\left(30 a^{\prime \prime}\right)^{-1}\left(32 a^{\prime \prime}\right)^{-1}\left(34 a^{\prime \prime}\right)^{1}(0.51)$ \\
$14^{2} A^{\prime \prime}$ & 25.639 & $\left(31 a^{\prime}\right)^{-1}\left(29 a^{\prime}\right)^{-1}\left(34 a^{\prime \prime}\right)^{1}(-0.45)$ \\
& & $\left(31 a^{\prime}\right)^{-1}\left(32 a^{\prime \prime}\right)^{-1}\left(33 a^{\prime}\right)^{1}(0.71)$ \\
& & $\left(30 a^{\prime \prime}\right)^{-1}\left(32 a^{\prime \prime}\right)^{-1}\left(34 a^{\prime}\right)^{1}(-0.46)$ \\
\hline \hline & &
\end{tabular}

in this work have been performed with the cc-pVTZ basis set. Table II lists the VIEs of molecular orbitals obtained with the SACCI cc-pVTZ, EPT-P3+ aug-cc-pVTZ and the EPT-OVGF aug-cc-pVTZ methods.

The experimental VUV PES of FNMA is presented in Fig. 2 (red line) together with the VIE values, reported as bars, calculated with the OVGF, P3+, and SACCI methods. A simulated spectrum (blue dash-dotted line) is also displayed in Fig. 2. It was produced using Gaussian line profiles of $0.18-\mathrm{eV}$ FWHM, which corresponds to the experimental energy resolution, and the VIEs and intensities obtained at the SACCI level. The observed experimental peak broadening is significantly larger than the total broadening expected from the photon and spectrometer resolutions. The unresolved vibrational structure is therefore mainly responsible for the observed broadening of the spectral components. Because of this broadening and the relatively high cation state density in some energy regions, groups of states have been merged into single peak positions, which are indicated in the figure by black stars. The VIEs corresponding to these peak positions are listed in Table II, where they are compared with the calculated values obtained at different levels of theory.

\section{B. Resonant Auger spectra}

The most prominent features in RA spectra are associated with cationic states in $1 h$ (participator) and $2 h-1 p$ (spectator) configurations. The participator states are similar to those populated upon direct valence ionizations and their VIEs from the neutral ground state are presented in Table II. The VIEs of the spectator states, which can be populated upon RA decay 


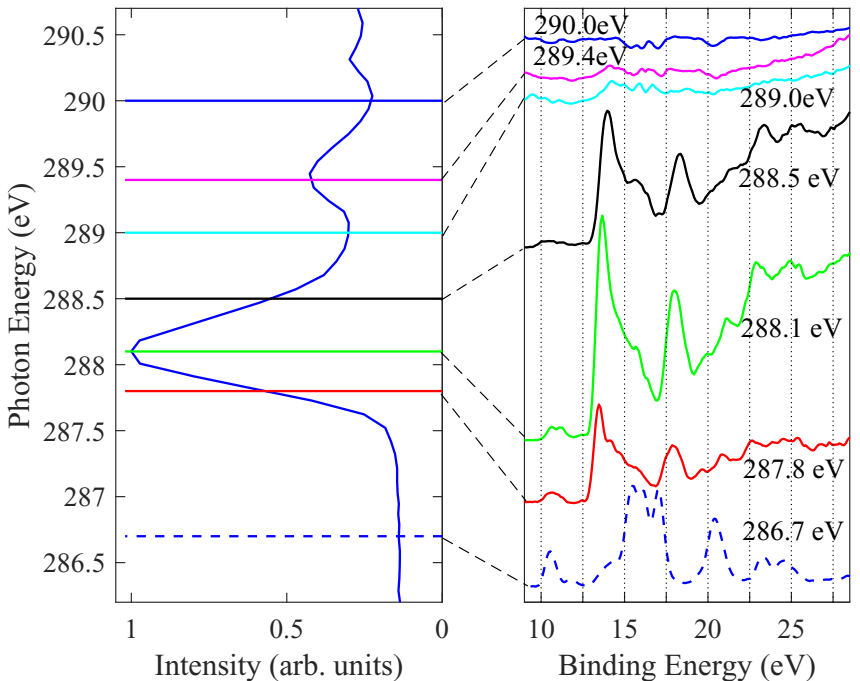

FIG. 3. Shown on the right is the C $1 s$-edge RA spectra of FNMA recorded at the excitation energies indicated by the horizontal lines in the NEXAFS spectrum on the left. The below-resonance contribution (blue dashed line) has been removed from the RA spectra. The experimental energy resolution of the NEXAFS spectrum is $\sim 30 \mathrm{meV}$ and of the RA spectra $\sim 450 \mathrm{meV}$.

of FNMA, have been obtained with the SACCI-R-general cc-pVTZ method and are listed in Table III.

Figures 3-5 show the RA spectra (right panel) for the $\mathrm{C} 1 s, \mathrm{~N} 1 s$, and $\mathrm{O} 1 s$ edges, respectively. Their respective NEXAFS spectra (left panel), with the excitation energies of the RA spectra marked, are also shown in the figures. In order to directly compare the results at different edges and resonances within an edge, the spectra associated with the main resonances are plotted together in Fig. 6, which also shows our peak assignments. Resonant contributions have been highlighted in these figures by subtracting the belowresonance spectrum from all RA spectra. The experimental

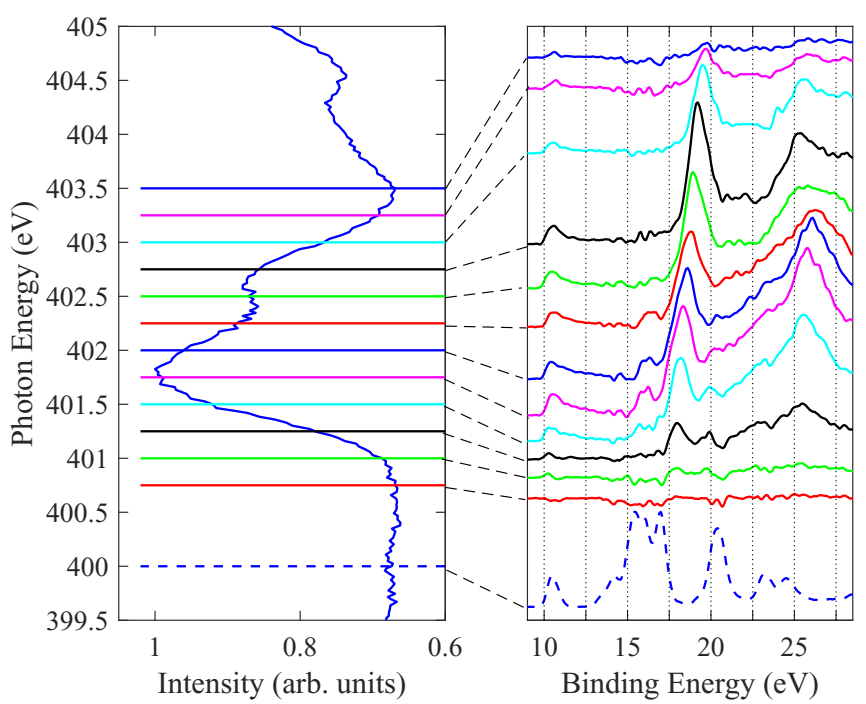

FIG. 4. Same as Fig. 3 but for the N $1 s$ edge. The experimental energy resolution of the NEXAFS spectrum is $\sim 70 \mathrm{meV}$ and of the RA spectra $\sim 450 \mathrm{meV}$.

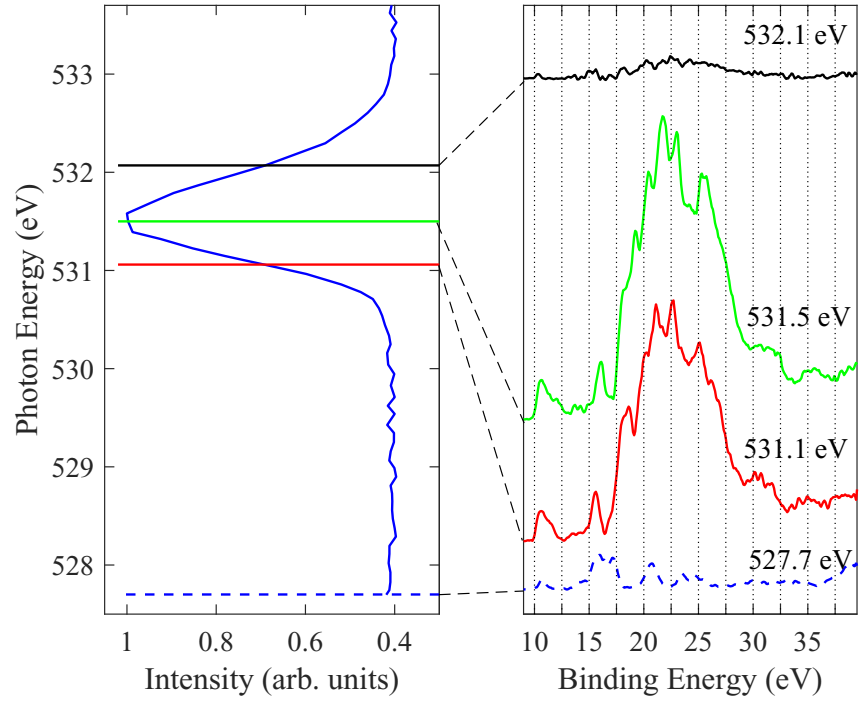

FIG. 5. Same as Fig. 3 but for the O $1 s$ edge. The experimental energy resolution of the NEXAFS spectrum is $\sim 70 \mathrm{meV}$ and of the RA spectra $\sim 600 \mathrm{meV}$.

resolution of the NEXAFS and RA spectra is specified in the figure captions.

Figure 6 reveals significant differences in the RA spectra between the three edges. The probability of participator Auger decay is related to the spatial overlap between the $1 s$ orbital of the core-excited site, the excited-electron orbital [the lowest unoccupied molecular orbital (LUMO) or the higher-lying orbital above the LUMO], and the $1 h$ orbital created after Auger decay $[1,38]$. The observed differences can be qualitatively explained using the spatial distribution of the orbitals shown in Fig. 7 and the calculated VIEs in Table II.

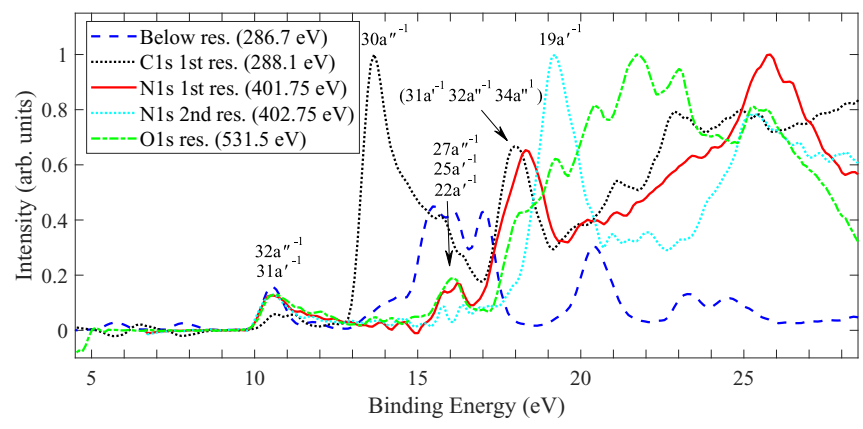

FIG. 6. The RA spectra of the main resonances for the C $1 s$, $\mathrm{N} 1 s$, and $\mathrm{O} 1 s$ edges and the below-resonance spectrum recorded at a photon energy of $286.7 \mathrm{eV}$. The below-resonance contribution has been removed from the RA spectra. The RA spectra have been normalized to 1 at their maximum values and the below-resonance spectrum is normalized to the $\mathrm{C} 1 s$-edge RA spectrum. The belowresonance excitation corresponds to a direct photoionization process and thus the peak positions of the blue dashed line indicate where the participator Auger peak positions are expected in the RA spectra. Peak assignments of the RA spectra are indicated by the configurations of the states. The experimental energy resolutions of the RA spectra for the different edges are specified in the respective captions of Figs. 3-5. 


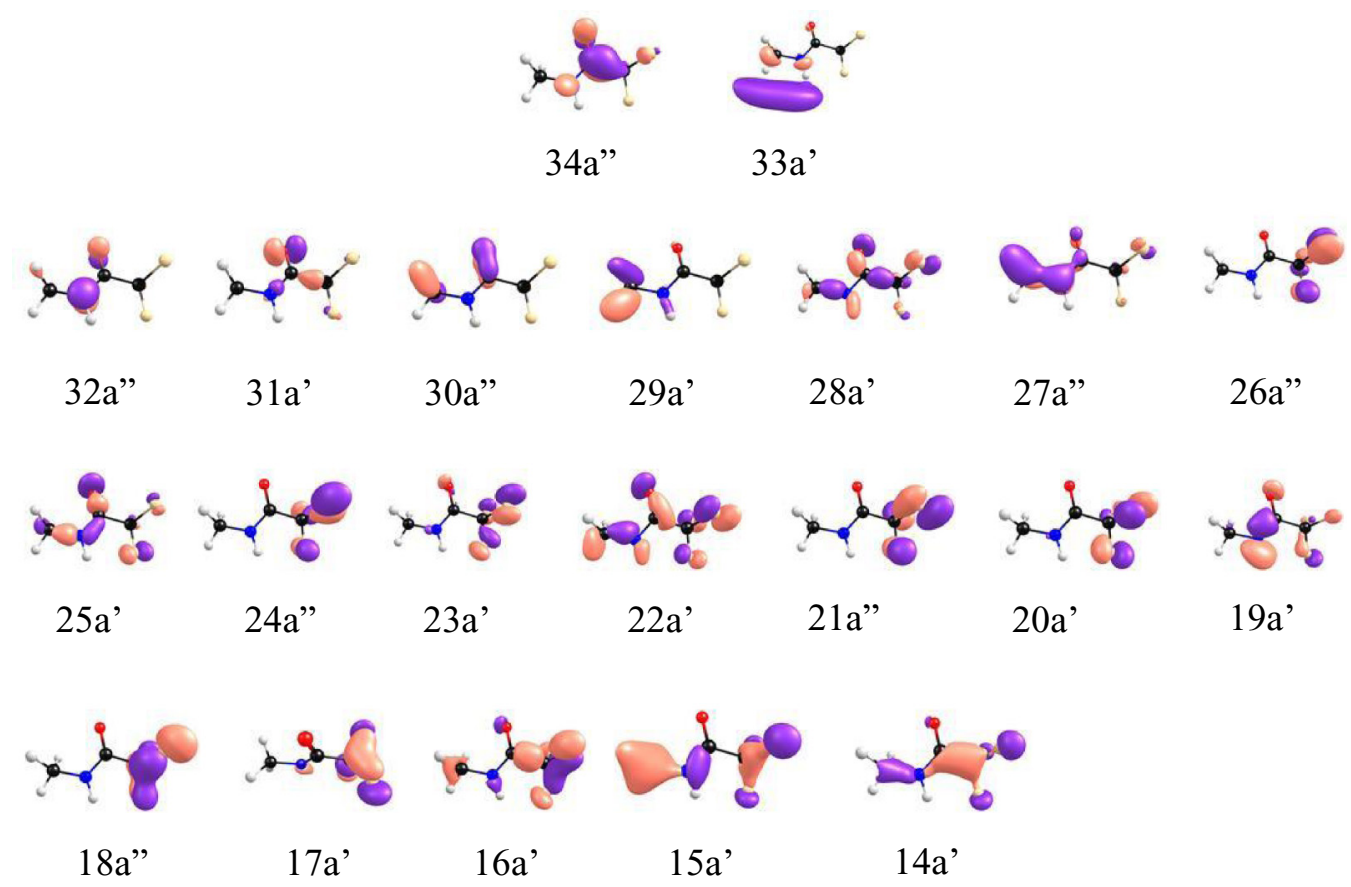

FIG. 7. Molecular orbitals of the FNMA ground-state geometry which was optimized at the CAM-B3LYP-3BJ aug-cc-pVTZ level of theory. The two different colors of the molecular orbitals correspond to different signs of the wave functions. The color code for atoms can be found in Fig. 1. The $33 a^{\prime}$ and $34 a^{\prime \prime}$ orbitals are virtual.

The lowest-energy peak at $10.5 \mathrm{eV}$ in the $\mathrm{C} 1 s$-edge RA spectrum at the excitation energy of $288.1 \mathrm{eV}$ has the lowest intensity compared to other RA spectra presented in Fig. 6. We note that the $\mathrm{C} 1 s$ site in FNMA has three inequivalent carbon atoms with supposedly rather different $\mathrm{C} 1 s$ electron binding energies. In our previous work [32] the 288.1-eV resonance was assigned to the promotion of the $1 s$ core electron at the $\mathrm{C} 2$ atom to the $\pi^{*}$ antibonding molecular orbital which resembles the $34 a^{\prime \prime}$ orbital of the FNMA ground state (Fig. 7). Following the peak assignment of the VUV PES spectrum, the $10.5-\mathrm{eV}$ feature is attributed to ionization of the $31 a^{\prime}$ and $32 a^{\prime \prime}$ orbitals. Since these orbitals have small contributions from the C2 atom, the intensity of the corresponding Auger peak is low. On the other hand, the $\mathrm{C} 1 s$-excited RA spectrum possesses a pronounced peak at $13.7 \mathrm{eV}$. The high relative intensity of this peak can be explained by the fact that the corresponding ionized orbital, $30 a^{\prime \prime}$, exhibits significant density at the $\mathrm{C} 2$ atom. By the same reasoning we can explain the absence of the $30 a^{\prime \prime}$ feature in the two $\mathrm{N} 1 s$ RA spectra, since the $30 a^{\prime \prime}$ orbital has no significant contributions from the $\mathrm{N}$ atom. However, such an explanation does not hold for the $\mathrm{O} 1 s \mathrm{RA}$ spectrum, as it does not exhibit the $30 a^{\prime \prime}$ feature despite the fact that the $30 a^{\prime \prime}$ orbital has significant contributions from the $\mathrm{O}$ atom. This can be partly understood by comparing different features in the O $1 s$ RA spectrum rather than comparing the $30 a^{\prime \prime}$ feature across the different core-excited RA spectra. Among the $30 a^{\prime \prime}, 31 a^{\prime}$, and $32 a^{\prime \prime}$ orbitals, $31 a^{\prime}$ and $32 a^{\prime \prime}$ have strong contributions from the $\mathrm{O}$ atom, whereas $30 a^{\prime \prime}$ is diffuse and has predominantly $\pi(\mathrm{C}=\mathrm{O})$ bonding character. As such, the $31 a^{\prime}$ and $32 a^{\prime \prime}$ features in the O $1 s$ RA spectrum are relatively strong compared to $30 a^{\prime \prime}$.

The RA spectra of all edges display the common characteristic of small structures in the region of $15.3-16.8 \mathrm{eV}$, although it is less pronounced for the second $\mathrm{N} 1 s$ resonance. According to the theoretical VIEs (Table II), this binding energy (BE) range corresponds to the ionization of several molecular orbitals. Based on the molecular orbital shapes, the most likely ionized orbitals associated with these peaks are $27 a^{\prime \prime}, 25 a^{\prime}$, and $22 a^{\prime}$ since they all have significant density at the $\mathrm{C} 2, \mathrm{~N}$, and $\mathrm{O}$ atoms. Consequently, these orbitals are expected to generate peaks for all edges studied, which results in the common features.

According to the calculations (Table III), the first spectator Auger peak should have a BE of $\sim 19.3 \mathrm{eV}$. A pronounced peak is observed at $\sim 18 \mathrm{eV}$ for the $\mathrm{C} 1 s$ edge and for the first resonance $(401.75 \mathrm{eV})$ of the $\mathrm{N} 1 s$ edge. For the $\mathrm{O} 1 s$ edge a corresponding peak at $18 \mathrm{eV}$ is also discernible although slightly weaker. Since the position of the peak approximately matches the calculated VIE and because there is no participator Auger contribution expected in this BE region, we assign these peaks to the spectator Auger decay that produces the $8^{2} A^{\prime} 2 h-1 p$ final state. At higher BEs the RA intensity increases due to the multitude of spectator Auger states located in this region as shown in Table III.

The RA spectrum recorded at the $\mathrm{N} 1 s$ edge is shown in Fig. 4. It displays a shift of the peak attributed to spectator Auger decay located at $18.3 \mathrm{eV}$ upon excitation at the first resonance $(401.75 \mathrm{eV})$ to $19.2 \mathrm{eV}$ when exciting at the second resonance $(402.75 \mathrm{eV})$. Although it seems to be a shift of the same peak, the peak at $19.2 \mathrm{eV}$ cannot be attributed to spectator Auger state. The second NEXAFS resonance $(402.75 \mathrm{eV})$ involves core-electron excitation to the $33 a^{\prime}$ orbital [32] and therefore the corresponding spectator state should have an electron at $33 a^{\prime}$. However, according to our theoretical predictions, the lowest-energy spectator state with such a characteristic has an electronic configuration of 
$\left(31 a^{\prime}\right)^{-1}\left(32 a^{\prime \prime}\right)^{-1}\left(33 a^{\prime}\right)^{1}$ with a VIE of $25.6 \mathrm{eV}$. As such, the experimental peak at $19.2 \mathrm{eV}$ cannot originate from the Auger spectator decay and we assign it to the participator decay leading to the final state that corresponds to ionization of the $19 a^{\prime}$ orbital. Indeed, as inferred from the assignment of the VUV spectrum, the ionization energy of the $19 a^{\prime}$ orbital is $19.6 \mathrm{eV}$. Further support for this assignment is provided by the FNMA molecular orbitals (Fig. 7). The large peak at $19.6 \mathrm{eV}$ is only observed upon excitation at the second $\mathrm{N}$ $1 s$-edge resonance (N $\left.1 s \rightarrow 33 a^{\prime}\right)$, but not at the first one $\left(\mathrm{N} 1 s \rightarrow 34 a^{\prime \prime}\right)$. The $33 a^{\prime}$ orbital differs from $34 a^{\prime \prime}$ by having a significant density at the $\mathrm{H}$ atom next to the $\mathrm{N}$ site (see specifically the excited-state orbitals of Fig. 4 in Ref. [32]). Since the $19 a^{\prime}$ orbital has also a large density at this $\mathrm{H}$ atom, the probability of the electron excited to the $33 a^{\prime}$ orbital to decay to $19 a^{\prime}$ is higher compared to that excited to $34 a^{\prime \prime}$.

In the RA spectra (Fig. 6) the $10.5-\mathrm{eV}$ peak exhibits a tail towards higher BE extending over several eVs. This tail is less pronounced for the below-resonance (Fig. 6) and VUV (Fig. 2) spectra. It suggests that, compared to prompt ionization, the core excitation and the subsequent Auger decay lead to the formation of different cation excited states. Since the two lowest states $1^{2} A^{\prime \prime}$ and $1^{2} A^{\prime}$ are close in energy and the next electronically excited state following them is $\sim 3 \mathrm{eV}$ away (Table II), it is most likely that the tail originates from vibrational excitation of $1^{2} A^{\prime \prime}$ and/or $1^{2} A^{\prime}$ cations. In order to get insight into which state is more prone to vibrational excitation upon resonant Auger decay, we refer to the FNMA molecular orbitals (Fig. 7). The $1^{2} A^{\prime \prime}$ state is associated with ionization of the $32 a^{\prime \prime}$ orbital. Since this orbital has a lone-pair character, i.e., does not contribute significantly to the molecular bonding, the $1^{2} A^{\prime \prime}$ state should have a similar geometry compared to the neutral FNMA. This implies a favorable Franck-Condon overlap between vibrational ground states of the neutral FNMA and the $1^{2} A^{\prime \prime}$ cation. In contrast, the $31 a^{\prime}$ orbital is not solely of the lone-pair character and favorable Franck-Condon overlap will occur for vibrationally excited states of the $1^{2} A^{\prime}$ cation. Based on this, we can conclude that the formation of $1^{2} A^{\prime}$ cations is enhanced upon the RA decays which in turn are created with vibrational excitation. We note that significant nuclear dynamics prior to the Auger decay may also result in a different vibrational excitation following RA decay compared with VUV ionization.

\section{Photoelectron-ion coincidence spectra}

Figures 8(a)-8(d) show the electron spectra (red dashed line) together with the ion mass spectra in which the ions (see the legend) have been detected in coincidence with the ejected electrons of a specific kinetic energy. The results are presented for the VUV ionization [Fig. 8(a)] and for the resonant excitation at the $\mathrm{C} 1 s$ [Fig. 8(b)], $\mathrm{N} 1 s$ [Fig. 8(c)], and $\mathrm{O} 1 s$ edge [Fig. 8(d)]. The ion-electron-coincidence measurements were performed for several electron kinetic energies, which are marked by black dots in Fig. 8. Through the selection of the kinetic energy of the electron we look at a selected subset of electron-ion coincidences, concentrating on single Auger decay in the outer valence energy range. In these figures the below-resonance contributions have not been removed. Figures $8(\mathrm{e})-8(\mathrm{~h})$ show the corresponding branching fractions, i.e., the fractional yield of an ion fragment with respect
TABLE IV. Calculated thermodynamic thresholds and experimental appearance energies upon VUV ionization for the fragmentation channels associated with the production of the specific ion fragments. The appearance energies are determined as the first point where the signal appears. A minimum error margin of $\pm 0.1 \mathrm{eV}$ is estimated based on the width $(2 \sigma)$ of the VUV PES peaks.

\begin{tabular}{lcc}
\hline \hline $\begin{array}{l}\text { Ion } \\
\text { fragment }\end{array}$ & $\begin{array}{c}\text { Thermodynamic } \\
\text { threshold (eV) }\end{array}$ & $\begin{array}{r}\text { Experimental appearance } \\
\text { energy (eV) }\end{array}$ \\
\hline $\mathrm{CH}_{3} \mathrm{NHCO}^{+}$ & 10.64 & 10.4 \\
$\mathrm{CH}_{2} \mathrm{NHCOCF}_{3}{ }^{+}$ & 11.69 & 13.4 \\
$\mathrm{CF}_{3}{ }^{+}$ & 12.80 & 15.1 \\
$\mathrm{CH}_{3} \mathrm{NHCOCF}_{2}{ }^{+}$ & 13.10 & 14.8 \\
$\mathrm{CH}_{3}{ }^{+}$ & 14.05 & 14.8 \\
$\mathrm{CF}_{2} \mathrm{CO}^{+}$ & 14.41 & 14.4 \\
$\mathrm{NHCH}_{2}{ }^{+}$ & 15.54 & 16.8 \\
$\mathrm{NH}^{+}$ & 21.26 & \\
\hline \hline
\end{tabular}

to the total yield of all plotted fragments. Thermodynamic threshold energies associated with the displayed ion-fragment channels are indicated by vertical lines. The thermodynamic thresholds and the experimental appearance energies are listed in Table IV. The appearance energy is determined as the first point where the signal emerges and the lowest limit of the error margin of $\pm 0.1 \mathrm{eV}$ is the estimated experimental resolution that corresponds to $\pm \sigma$ of the VUV PES peaks.

We first concentrate on the valence ionization [Figs. 8(a) and 8(e)]. The spectra were recorded at a photon energy of $60 \mathrm{eV}$ and with the experimental energy resolution specified in the figure caption. The intensity curve for the parent ion (blue solid line) has a peak close to the $10.5-\mathrm{eV}$ feature of the VUV PES. The electrons ejected with higher kinetic energies are associated with higher-energy excited states of cations implying that the cations possess excess energy which can eventually lead to fragmentation. According to the theoretical predictions, the lowest-energy dissociation channel involves detachment of $\mathrm{CF}_{3}$ and lies $0.09 \mathrm{eV}$ above the first VIE value calculated with the same method (CAM-B3LYPD3BJ aug-cc-pVTZ). This theoretical value is in reasonable agreement with the experimental appearance energy of the $\mathrm{CH}_{3} \mathrm{HNCO}^{+}$fragment, which is $10.4 \pm 0.1 \mathrm{eV}$ [green solid curve in Fig. 8(a)]. The branching fractions presented in Fig. 8(e) show that the $\mathrm{CH}_{3} \mathrm{HNCO}^{+}$ion competes with the parent ion and as soon as the $\mathrm{CH}_{3} \mathrm{HNCO}^{+}+\mathrm{CF}_{3}$ channel opens, the importance of the parent ion is gradually diminishing until it nearly disappears at higher electron kinetic energies. Opening of this fragmentation channel has two possible causes. One scenario implies that it is connected with the first electronically excited state $1^{2} A^{\prime}$, which has the $\left(31 a^{\prime}\right)^{-1}$ electronic configuration. On the other hand, it can originate from the vibrationally excited ground state $1^{2} A^{\prime \prime}$ though, as elaborated in the preceding section, significant vibrational excitation of the $1^{2} A^{\prime \prime}$ cationic state is unlikely.

The large ions appearing next are those formed by detachment of the $\mathrm{H}$ [red solid line in Fig. 8(a)] and F [black solid line in Fig. 8(a)] atoms, which have appearance energies of 13.4 and $14.8 \mathrm{eV}$, respectively. The smaller fragments detected in coincidence with photoelectrons are shown by dash-dotted lines in Fig. 8 (see the legend). Their appearance 

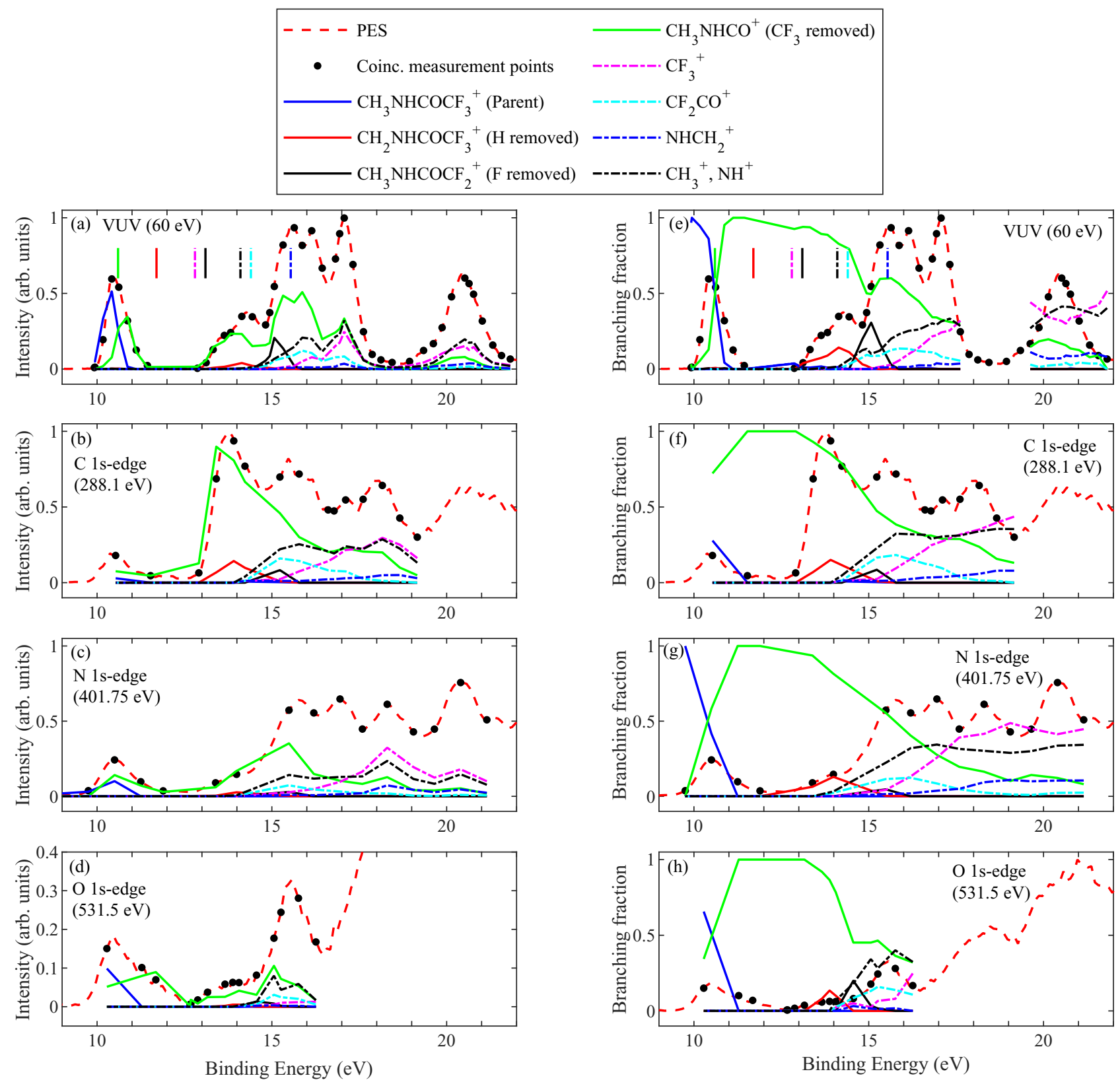

FIG. 8. (a)-(d) Plots showing the electron spectra (red dashed lines) of FNMA and the intensities of fragment ions (see the legend) in coincidence with the ejected electrons. The plots are shown for (a) the VUV (60-eV) valence ionization and for the resonant excitations to the $34 a^{\prime \prime}$ orbital at the (b) C $1 s(288.1-\mathrm{eV})$, (c) N $1 s(401.75-\mathrm{eV})$, and (d) O $1 s(531.5-\mathrm{eV})$ edges. The experimental energy resolutions of the electron spectra are $\sim 220 \mathrm{meV}$ (VUV PES), $\sim 450 \mathrm{meV}$ (RA at the $\mathrm{C} 1 s$ edge), $\sim 500 \mathrm{meV}$ (RA at the N $1 s$ edge), and $\sim 600 \mathrm{meV}$ (RA at the O $1 s$ edge). The black dots on the VUV PES and RA curves mark the kinetic energies of electrons that were measured in coincidence with ions. The VUV PES and RA spectra have been normalized to 1 at the maximum intensity and the coincident ion intensities have been normalized to the PES peak at $10.5 \mathrm{eV}$. Below-resonance contributions have not been removed. The fragment ions with mass equal to $28 \mathrm{u}\left(\mathrm{NHCH}^{+}\right.$and $\mathrm{CO}^{+}$) have been omitted due to contributions from $\mathrm{N}_{2}{ }^{+}$for the $\mathrm{N} 1 s$ edge. (e)-(h) Branching fractions, i.e., the fractional yield with respect to that of the sum of all displayed fragment ions, are shown together with the VUV PES and RA spectra (red dashed lines). The VUV branching fractions have been omitted in the region of $\mathrm{BE}$ equal to $17.6-19.6 \mathrm{eV}$ in (e) due to the limited signal resulting from a low cross section for the photoelectron process. The vertical lines mark the thermodynamic threshold energies of the fragment ions using the line styles and colors shown in the legend. The statistical uncertainty of the branching fraction is less than 0.05 (rms).

energies lie in the range of 14-17 eV. By studying the branching fractions presented in Fig. 8(e), one observes that all curves are continuous after the corresponding fragmentation channels have emerged except the channels associated with detachment of $\mathrm{H}$ (red solid line) and $\mathrm{F}$ (black solid line), which just display a single peak. A plausible explanation for such a phenomenon can be given based on comparison of the thermodynamic thresholds of the fragmentation channels and the experimental appearance energies (Table IV). According to our calculations, the thresholds to detach the $\mathrm{H}$ and $\mathrm{F}$ 
atom are 11.69 and $13.10 \mathrm{eV}$, respectively, whereas the appearance energies are 13.4 and $14.8 \mathrm{eV}$, respectively. Such substantial differences imply that upon ejection of the $\mathrm{H}$ or $\mathrm{F}$ atom, the $\mathrm{CH}_{2} \mathrm{NHCOCF}_{3}{ }^{+}$or $\mathrm{CH}_{3} \mathrm{NHCOCF}_{2}{ }^{+}$fragment has an internal energy of more than $1.5 \mathrm{eV}$. As the higherenergy excited states are populated (i.e., electrons with lower kinetic energies are ejected), the excess energy grows such that the $\mathrm{CH}_{2} \mathrm{NHCOCF}_{3}{ }^{+}$and $\mathrm{CH}_{3} \mathrm{NHCOCF}_{2}{ }^{+}$fragments can eventually undergo further fragmentation. For example, $\mathrm{CH}_{2} \mathrm{NHCOCF}_{3}{ }^{+}$might break-up into $\mathrm{CH}_{2} \mathrm{NHCO}$ and $\mathrm{CF}_{3}{ }^{+}$. Indeed, the disappearance of $\mathrm{CH}_{2} \mathrm{NHCOCF}_{3}{ }^{+}$coincides with the emergence of the $\mathrm{CF}_{3}{ }^{+}$fragment [see the red solid and purple dash-dotted curves around $15 \mathrm{eV}$ in Fig. 8(e)]. This explanation is also valid for the behavior of the $\mathrm{CH}_{3} \mathrm{NHCO}^{+}$ fragment. For this fragment the thermodynamic threshold and appearance energy are similar, implying that it forms with almost no internal energy, i.e., all the excess energy of the parent ion fragmenting into the $\mathrm{CH}_{3} \mathrm{NHCO}^{+}+\mathrm{CF}_{3}$ channel will be transferred into the kinetic energy of the fragments preventing secondary fragmentation.

The core-excited coincidence spectra shown in Figs. 8(b) $-8($ d) and the corresponding branching fractions in Figs. 8(f)-8(h) reveal appearance energies similar to those for the VUV excitation. However, for the resonant Auger decays the measurements were performed at fewer electron kinetic energies compared to the VUV experiment, which makes determination of appearance energies less accurate. As can be seen from Figs. 8(e)-8(h), for excited states associated with BEs up to $16-17 \mathrm{eV}$, the removal of $\mathrm{CF}_{3}$ is the most probable fragmentation channel. At higher BEs the decay routes corresponding to breakup into smaller fragments become dominant for the core excitations, similar to the case of VUV ionization.

Generally, the branching fractions for all excitations are very similar, i.e., at a specific $\mathrm{BE}$ (or excess energy of the parent ions) we do not observe any significant effect of the local character of the core excitations. Such site-specific effects have however been observed for other molecules by studying the fragmentation yields in a small internal energy range $[18,30]$.

In our previous studies of FNMA fragmentation induced by core-valence excitations, different fragmentation patterns were observed [32]. The results of the present work employing the Auger electron-ion-coincidence technique are essential to understand the observations reported in that work. In particular, the ion fragment formed by detachment of the $\mathrm{CF}_{3}$ moiety was found to be significantly enhanced upon excitation of the first resonance of the $\mathrm{C} 1 s$ edge. The present study explains this observation by the much larger RA peak at a BE of $13.7 \mathrm{eV}$ upon excitation at the $\mathrm{C} 1 s$ edge compared with the other excitations. As assigned earlier in this work, this peak represents the ionization of the $30 a^{\prime \prime}$ orbital. As shown in Fig. 7, this orbital has a $\pi \mathrm{C}=\mathrm{O}$ bonding character, which includes the $\mathrm{C} 2$ atom. Thus, the overlap with the $\mathrm{C} 21 s$ orbital is large and this results in a pronounced RA peak. The peak at $13.7 \mathrm{eV}$ mostly has contributions from $\mathrm{CF}_{3}$ detachment [green solid line in Fig. 8(b)], while the $\mathrm{C}=\mathrm{O}$ bond stays intact. Since the available energy created by removing an electron from the $30 a^{\prime \prime}$ orbital is not sufficient for breaking the $\mathrm{C}=\mathrm{O}$ bond, the weak $\mathrm{CH}_{3} \mathrm{NHCO}-\mathrm{CF}_{3}$ bond is instead broken.
The spectator Auger decay process is different from the participator Auger decay in the sense that the resonantly excited electron stays in the orbital to which it was excited during the Auger decay. Hence, a stronger correlation could be envisaged between the antibonding character of, e.g., the $34 a^{\prime \prime}$ orbital and the breaking of the bonds displaying antibonding character. However, the spectator Auger feature at $\sim 18 \mathrm{eV}$ reveals no significant enhancement of a specific ion fragment [Figs. 8(f) and 8(g)]. For example, the peaks on either side of the spectator Auger feature (i.e., at $\sim 17$ and $20 \mathrm{eV}$ ) in the RA spectrum for the $\mathrm{N} 1 s$ edge [Fig. 8(g)] are mainly associated with the formation of a $1 h$ final state. Although the spectator Auger final state has a different configuration $(2 h-1 p)$, there is no clear change in ion branching fraction correlated to this state.

More generally, when considering all studied binding energies in Figs. 8(e)-8(h), which mainly correspond to participator Auger states, we do not observe any significant correlation of the ion branching fractions to the Auger peaks, i.e., the branching fractions are rather structureless functions of binding energies and appearing of different channels can be explained in terms of their thermodynamic thresholds, as discussed above. Thus, the lack of significant correlation between the fragmentation patterns and Auger final states suggests that the amount of excess energy the molecule gains upon RA decay is the dominant factor for how it fragments. We note, however, that preferential breaking of specific bonds with respect to the character of the orbitals involved in the Auger process has been demonstrated in other molecules [13].

\section{CONCLUSION}

In this work, we have presented VUV PES and RA spectra of FNMA measured at the $\mathrm{C} 1 s, \mathrm{~N} 1 s$, and $\mathrm{O} 1 s$ edges as well as results of RA electron-ion-coincidence measurements. With the help of the theoretical calculations performed for cationic states of $1 h$ and $2 h-1 p$ configurations, we have assigned spectral features in the recorded VUV PES and RA spectra. Significant differences between the RA spectra recorded upon site-specific $\mathrm{C} 1 s^{-}, \mathrm{N} 1 s$-, and $\mathrm{O} 1 s$-edge excitation and upon state-specific excitation were observed. These differences reflect contrasting Auger populations, which to a high degree can be rationalized by the distinct overlaps of the molecular orbitals directly involved in the Auger process [38]. The Auger population is found to strongly influence the fragmentation pattern of the molecule, in agreement with previous studies $[13,28]$.

By correlating a specific Auger final state with its fragmentation channels we obtained further understanding of how the fragmentation depends on the Auger decay and site-specific excitation. For example, we could explain our previous results [32] that demonstrated a significantly higher yield, i.e., far above the statistical uncertainty, of the ion formed by detaching $\mathrm{CF}_{3}$ upon excitation of the first $\mathrm{C} 1 s$-edge resonance compared with those at the $\mathrm{N} 1 s$ and $\mathrm{O} 1 s$ edges. Also, we examined the participator and spectator RA decay with respect to differences in fragmentation. This was motivated by their potentially different capacity for selective bond breaking. Since the spectator decay produces a final state in which the core-excited electron occupies a virtual molecular orbital that can be of antibonding character, the nature of this orbital may 
cause a selective bond breaking. However, our results show no significant change of the ion branching fractions correlated with the spectator Auger state involving the excitation to an antibonding orbital. Moreover, no significant correlation between the ion branching fractions and the Auger peaks was observed in general. The lack of significant correlation between the fragmentation patterns and Auger final states suggests that the amount of excess energy the molecule gains upon RA decay is the dominant factor for how it fragments.

\section{ACKNOWLEDGMENTS}

We acknowledge Elettra Sincrotrone Trieste for providing access to its synchrotron radiation facilities. Calculations were performed on resources at the Chalmers Centre for Computational Science and Engineering provided by the Swedish National Infrastructure for Computing. Support from the Stockholm-Uppsala Centre for Free Electron Laser Research is also acknowledged.
[1] D. B. Thompson, D. Ji, S. Y. Chen, and D. M. Hanson, J. Phys. B 32, 5711 (1999).

[2] F. Gelmukhanov and H. Ågren, Phys. Rep. 312, 87 (1999).

[3] G. B. Armen, H. Aksela, T. Åberg, and S. Aksela, J. Phys. B 33, R49 (2000).

[4] S. L. Sorensen and S. Svensson, J. Electron Spectrosc. Relat. Phenom. 114-116, 1 (2001).

[5] W. Eberhardt, T. K. Sham, R. Carr, S. Krummacher, M. Strongin, S. L. Weng, and D. Wesner, Phys. Rev. Lett. 50, 1038 (1983).

[6] M. C. Nelson, J. Murakami, S. L. Anderson, and D. M. Hanson, J. Chem. Phys. 86, 4442 (1987).

[7] A. Naves de Brito, R. Feifel, A. Mocellin, A. B. Machado, S. Sundin, I. Hjelte, S. L. Sorensen, and O. Björneholm, Chem. Phys. Lett. 309, 377 (1999).

[8] A. Naves de Brito, S. Sundin, R. R. Marinho, I. Hjelte, G. Fraguas, T. Gejo, N. Kosugi, S. Sorensen, and O. Björneholm, Chem. Phys. Lett. 328, 177 (2000).

[9] K. Ueda, M. Simon, C. Miron, N. Leclercq, R. Guillemin, P. Morin, and S. Tanaka, Phys. Rev. Lett. 83, 3800 (1999).

[10] P. Erman, A. Karawajczyk, E. Rachlew, M. Stankiewicz, and K. Y. Franzen, J. Chem. Phys. 107, 10827 (1997).

[11] P. Morin, M. Simon, C. Miron, N. Leclercq, E. Kukk, J. D. Bozek, and N. Berrah, Phys. Rev. A 61, 050701(R) (2000).

[12] X. J. Liu, G. Prümper, E. Kukk, R. Sankari, M. Hoshino, C. Makochekanwa, M. Kitajima, H. Tanaka, H. Yoshida, Y. Tamenori, and K. Ueda, Phys. Rev. A 72, 042704 (2005).

[13] P. Bolognesi, J. A. Kettunen, A. Cartoni, R. Richter, S. Tosic, S. Maclot, P. Rousseau, R. Delaunay, and L. Avaldi, Phys. Chem. Chem. Phys. 17, 24063 (2015).

[14] P. Salen, V. Yatsyna, L. Schio, R. Feifel, R. Richter, M. Alagia, S. Stranges, and V. Zhaunerchy, J. Chem. Phys. 144, 244310 (2016).

[15] Y.-S. Lin, C.-C. Tsai, H.-R. Lin, T.-L. Hsieh, J.-L. Chen, W.-P. Hu, C.-K. Ni, and C.-L. Liu, J. Phys. Chem. A 119, 6195 (2015).

[16] Y.-J. Chiang, W.-C. Huang, C.-K. Ni, C.-L. Liu, C.-C. Tsai, and W.-P. Hu, AIP Adv. 9, 085023 (2019).

[17] L. Schwob, S. Dörner, K. Atak, K. Schubert, M. Timm, C. Bülow, V. Zamudio-Bayer, B. von Issendorff, J. T. Lau, S. Techert, and S. Bari, J. Phys. Chem. Lett. 11, 1215 (2020).

[18] J. H. D. Eland, P. Linusson, M. Mucke, and R. Feifel, Chem. Phys. Lett. 548, 90 (2012).

[19] H. Iwayama, N. Sisourat, P. Lablanquie, F. Penent, J. Palaudoux, L. Andric, J. H. D. Eland, K. Bucar, M. Zitnik, Y.
Velkov, Y. Hikosaka, M. Nakano, and E. Shigemasa, J. Chem. Phys. 138, 024306 (2013).

[20] S. Nagaoka, H. Fukuzawa, G. Prümper, M. Takemoto, O. Takahashi, K. Yamaguchi, T. Kakiuchi, K. Tabayashi, I. H. Suzuki, J. R. Harries, Y. Tamenori, and K. Ueda, J. Phys. Chem. A 115, 8822 (2011).

[21] S. Nagaoka, G. Prümper, H. Fukuzawa, M. Hino, M. Takemoto, Y. Tamenori, J. Harries, I. H. Suzuki, O. Takahashi, K. Okada, K. Tabayashi, X.-J. Liu, T. Lischke, and K. Ueda, Phys. Rev. A 75, 020502(R) (2007).

[22] K. Le Guen, M. Ahmad, D. Céolin, P. Lablanquie, C. Miron, F. Penent, P. Morin, and M. Simon, J. Chem. Phys. 123, 084302 (2005).

[23] C. Miron, M. Simon, N. Leclercq, D. L. Hansen, and P. Morin, Phys. Rev. Lett. 81, 4104 (1998).

[24] A. Mocellin, K. Wiesner, S. L. Sorensen, C. Miron, K. Le Guen, D. Céolin, M. Simon, P. Morin, A. B. Machado, O. Björneholm, and A. Naves de Brito, Chem. Phys. Lett. 435, 214 (2007).

[25] H. Fukuzawa, G. Pruemper, X.-J. Liu, E. Kukk, R. Sankari, M. Hoshino, H. Tanaka, Y. Tamenori, and K. Ueda, Chem. Phys. Lett. 436, 51 (2007).

[26] E. Itälä, D. T. Ha, K. Kooser, M. A. Huels, E. Rachlew, E. Nõmmiste, U. Joost, and E. Kukk, J. Electron Spectrosc. Relat. Phenom. 184, 119 (2011).

[27] E. Kukk, D. T. Ha, Y. Wang, D. G. Piekarski, S. Diaz-Tendero, K. Kooser, E. Itälä, H. Levola, M. Alcami, E. Rachlew, and F. Martin, Phys. Rev. A 91, 043417 (2015).

[28] H. Levola, E. Itälä, K. Schlesier, K. Kooser, S. Laine, J. Laksman, D. T. Ha, E. Rachlew, M. Tarkanovskaja, K. Tanzer, and E. Kukk, Phys. Rev. A 92, 063409 (2015).

[29] E. Itälä, H. Levola, D. T. Ha, K. Kooser, E. Rachlew, and E. Kukk, J. Phys. Chem. A 120, 5419 (2016).

[30] S. Zagorodskikh, J. H. D. Eland, V. Zhaunerchyk, M. Mucke, R. J. Squibb, P. Linusson, and R. Feifel, J. Chem. Phys. 145, 124302 (2016).

[31] L. Inhester, B. Oostenrijk, M. Patanen, E. Kokkonen, S. H. Southworth, C. Bostedt, O. Travnikova, T. Marchenko, S. K. Son, R. Santra, M. Simon, L. Young, and S. L. Sorensen, J. Phys. Chem. Lett. 9, 1156 (2018).

[32] C. Li, P. Salen, V. Yatsyna, L. Schio, R. Feifel, R. Squibb, M. Kamiska, M. Larsson, R. Richter, M. Alagia, S. Stranges, S. Monti, V. Carravetta, and V. Zhaunerchyk, Phys. Chem. Chem. Phys. 18, 2210 (2016).

[33] R. R. Blyth, R. Delaunay, M. Zitnik, J. Krempasky, R. Krempaska, J. Slezak, K. C. Prince, R. Richter, M. Vondracek, R. Camilloni, L. Avaldi, M. Coreno, G. Stefani, C. Furlani, M. 
De Simone, S. Stranges, and M. Y. Adam, J. Electron Spectrosc. Relat. Phenom. 101, 959-964 (1999).

[34] O. Plekan, M. Coreno, V. Feyer, A. Moise, R. Richter, M. De Simone, R. Sankari, and K. C. Prince, Phys. Scr. 78, 058105 (2008).

[35] https://webbook.nist.gov/cgi/cbook.cgi?ID=C7732185\&Mask $=20$
[36] M. J. Frisch et al., GAUSSIAN16, revision B.01 (Gaussian, Inc., Wallingford, 2016).

[37] H. H. Corzo, J. M. Krosser, A. Galano, and J. V. Ortiz, Theor. Chem. Acc. 135, 236 (2016).

[38] S. Stranges, M. Y. Adam, C. Cauletti, M. De Simone, C. Furlani, M. N. Piancastelli, P. Decleva, and A. Lisini, J. Chem. Phys. 97, 4764 (1992). 